\title{
Carcinoma Arising in Bladder Diverticulum
}

National Cancer Institute

\section{Source}

National Cancer Institute. Carcinoma Arising in Bladder Diverticulum. NCI Thesaurus. Code C160158.

A carcinoma arising in a bladder diverticulum. Approximately one-third to half of the cases represent non-invasive, low-grade or high-grade urothelial carcinomas. Approximately half of the invasive carcinomas are urothelial. The rest include adenocarcinomas, squamous cell carcinomas, and small cell carcinomas. 